\title{
Minority carrier barrier heterojunctions for improved thermoelectric efficiency
}

\author{
Peter G. Burke, ${ }^{1}$ Benjamin M. Curtin, ${ }^{2}$ John E. Bowers, ${ }^{1,2}$ Arthur C. Gossard ${ }^{1}$ \\ ${ }^{1}$ Materials Department, University of California, Santa Barbara \\ ${ }^{2}$ Electrical and Computer Engineering Department, University of California, Santa Barbara
}

\begin{abstract}
We propose and demonstrate the beneficial use of minority carrier blocking layers to lessen bipolar conduction at elevated temperatures and increase the Seebeck coefficient in a thermoelectric material. Bipolar conduction, caused by thermally activated intrinsic carriers in semiconductors, causes a detrimental "roll-over" in the Seebeck coefficient and increase in thermal conductivity, measured as a function of temperature. We propose that these negative effects can be lessened or delayed in a small band gap host matrix by incorporating wider band gap semiconductor layers to impede the thermal diffusion of minority carriers over majority carriers. To prove this concept, a composite material grown by molecular beam epitaxy of p-type InAs layers and electron blocking p-type AlSb layers was grown and measured. We show improved thermoelectric properties over just p-type InAs in measurements from $110 \mathrm{~K}-600 \mathrm{~K}$.
\end{abstract}




\section{Keywords}

Thermoelectrics; Heterojunction barrier; Molecular beam epitaxy; Unipolar

\section{Introduction}

The most common contribution to the inefficient use of energy is generation of waste heat. ${ }^{1}$ Waste heat is energy that escapes from a system without contributing to the work done. A device capable of capturing that waste heat and turning it back into electricity would inherently increase the efficiency of the system and lower net power consumption. Often the environment for the most effective heat capture demands that the unit be small, clean, and reliable. It is here, in the niche of energy harvesting, where thermoelectric materials can be of great use. ${ }^{2}$ The thermoelectric efficiency of a material is often ranked by the thermoelectric figure of merit, $z T$, consisting of the Seebeck coefficient, $S$, electrical conductivity, $\sigma$, and thermal conductivity, $\kappa$, by $z T=S^{2} \sigma T / \kappa$. Therefore, the thermoelectric efficiency of a material is improved by increasing its Seebeck coefficient and electrical conductivity and by decreasing its thermal conductivity. However, these parameters are often directly related to each other. Generally for a semiconductor, as the carrier concentration is increased, the electrical conductivity increases but unfortunately, the Seebeck coefficient decreases and the thermal conductivity increases as well. In addition, these parameters are temperature dependent. For most semiconductors, the Seebeck coefficient increases with temperature until it reaches a maximum and begins to decrease. The Seebeck coefficient can even change sign at very high temperature. The reduction in Seebeck coefficient is caused by the thermal activation of intrinsic carriers. When there is mixed n-type 
and p-type conduction, i.e. bipolar conduction, the negative and positive Seebeck coefficients of each, respectively, cancel each other out. Essentially, both negative charges and positive charges are present within the same material and diffuse along the thermal gradient. In this case, there is no net charge transfer and the Seebeck coefficient is zero.

Conventionally, the temperature at which thermally excited minority carriers cause a decrease in Seebeck coefficient can be raised by using a material with a wider band gap, or by increasing the extrinsic doping. ${ }^{3,4}$ In this work, we predict and demonstrate increasing the Seebeck coefficient at higher temperature by utilizing minority carrier blocking layers. By impeding the thermal diffusion of minority carriers, we push the scenario closer to the optimum single-carrier Seebeck coefficient and improve the thermoelectric efficiency. The concept of minority carrier blocking layers is implemented here with wide band gap AlSb layers on narrow band gap InAs layers, grown by molecular beam epitaxy (MBE), but is generally applicable to any combination of semiconductors where the heterojunction can block the flow of the minority carriers but not block the flow of majority carriers.

For example, the concept of confining or blocking charge carriers has been used extensively in semiconductor research before, indeed it is the basis for many optical devices such as solidstate lasers, LEDs, and detectors. Electron- and hole-blocking layers have been used for $\mathrm{PbIbN}^{5,6}$ and $\mathrm{nBn}^{7}$ infrared detectors to reduce dark current as compared to conventional PIN detectors. This results in an increase in detectivity and higher operating temperatures.

Recently, in collaboration with this paper, Bahk and Shakouri have extensively modeled the prospects of reducing the thermal conductivity of PbTe through minority carrier blocking by heterjunctions. ${ }^{8}$ In both cases, minority carrier blocking shows great promise for increasing zT through improving the Seebeck coefficient and reducing the thermal conductivity. In other examples of unique heterojunctions which can improve thermoelectric efficiency, Tynell et al. ${ }^{9}$ have created a superlattice of conductive oxides $(\mathrm{ZnO})$ and organic compounds (hydroquinone) and seen significant reductions in thermal conductivity. ${ }^{9}$ 


\section{Two-carrier temperature-dependent Seebeck coefficient}

The Seebeck coefficient for a material with one type of carrier increases as the Fermi level moves towards the center of the semiconductor band gap. This occurs for semiconductors at high temperature or low doping as determined in the non-degenerate case by Equation 1.

$$
S_{n, p}= \pm \frac{k_{B}}{e}\left(r+\frac{5}{2}+\ln \frac{2\left(2 \pi m_{n, p}^{*} k_{B} T\right)^{3 / 2}}{h^{3} n, p}\right)(\mathrm{V} / \mathrm{K}) \quad \text { Equation } 1
$$

where $m^{*}{ }_{n, p}$ is the effective mass of electrons or holes, $r$ is the scattering parameter, $k_{B}$ is the Boltzmann's constant, $h$ is the Planck's constant, $e$ is the charge on an electron, and $n$ and $p$ are the electron and hole concentrations. It is important to note that the Seebeck coefficient is negative for electrons and positive for holes, and can therefore go to zero or change sign when there is mixed n-type and p-type conduction. As shown in Equation 2, the net Seebeck coefficient, $S_{T}$, is a combination of the negative Seebeck coefficient, $S_{n}$, and the positive Seebeck coefficient, $\mathrm{S}_{\mathrm{p}}$, weighted by the electron mobility, $\mu_{n}$, electron concentration, hole mobility, $\mu_{p}$, and hole concentration.

$$
S_{T}=\frac{S_{n} \mu_{n} n+S_{p} \mu_{p} p}{\mu_{n} n+\mu_{p} p} \quad \text { Equation } 2
$$

As mentioned previously, when designing a thermoelectric material for use at high temperature, there have only been two options to overcome problems with intrinsic carrier activation: use a material with a wide band gap or increase the extrinsic doping. Wide band gap semiconductors are better at high temperature because the thermally activated intrinsic carrier density depends exponentially on the band gap energy. Higher extrinsic doping helps because it more heavily weights the majority carrier Seebeck coefficient, as demonstrated in Equation 2. However, if you could add minority carrier blocking layers, and essentially reduce the mobility of the minority carrier, this would lighten the effect of the minority carrier Seebeck coefficient and improve the total Seebeck coefficient.

Figure 1a describes a typical $n$-type thermoelectric material, where the Fermi level is close to the conduction band edge and the temperature gradient has established an electron concentration gradient from the hot side to the cold side. Figure $1 \mathrm{~b}$ shows the scenario where the same material is being used at higher temperatures, and thermally activated carriers are dominating the material's Seebeck coefficient. The Fermi level is positioned near the middle of the energy gap and electrons and holes are both present in appreciable concentrations. There is very little net 
charge difference between the hot and cold side. Figure 1c demonstrates the usefulness of a wide-band gap hole blocking region in an n-type thermoelectric. The holes cannot flow from the hot to the cold side and an appreciable net Seebeck coefficient is maintained. Figure 1d shows similar benefits for using a wide-band gap electron blocking region in a p-type thermoelectric.

a)

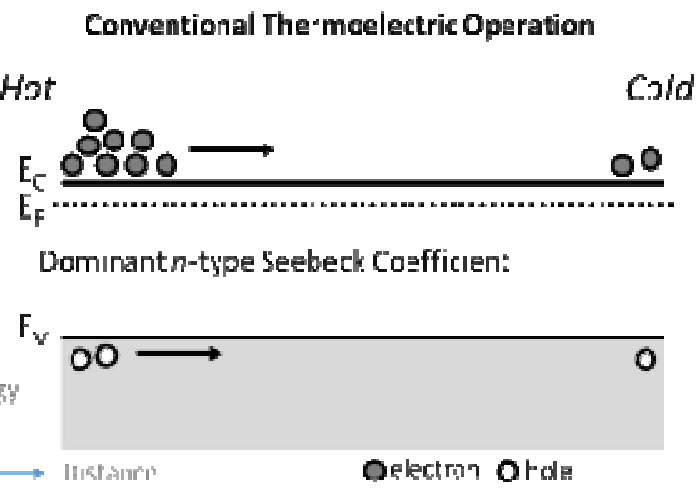

b)

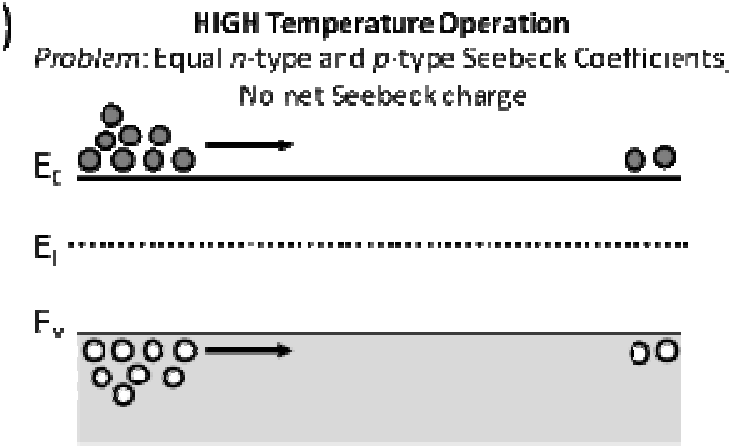

HIGH TemporatureOperat on

Solutior: Block the ninorit; chare catrierta maintain majority co vare carrier Seetreck Coetticient

c)

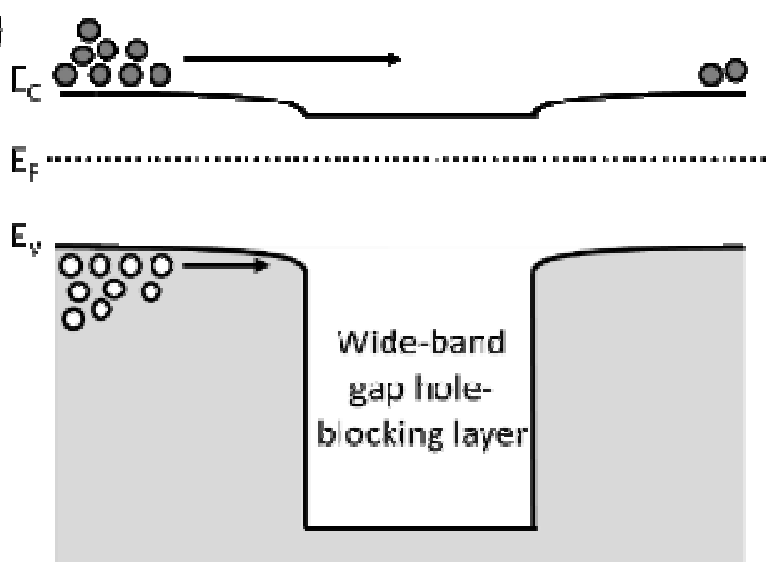

d)

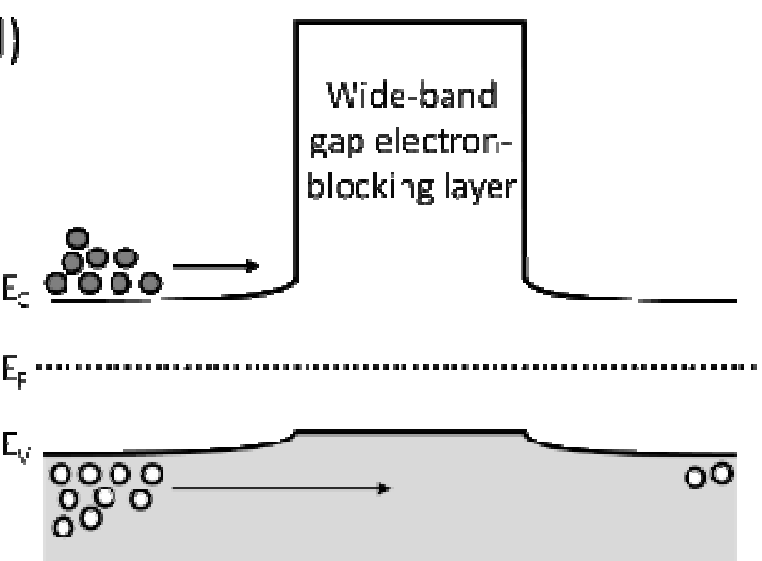

Figure 1. (a) Energy band diagram for a conventional thermoelectric material. (b) Energy band diagram for the same material operating at a much higher average temperature, where there is an appreciable concentration of minority carriers present due to thermal activation of electrons across the band gap. This scenario shows very low net charge difference between the hot side and cold side. (c) and (d) Energy band diagrams for hole-blocking and electronblocking regions to enhance $n$-type and $p$-type thermoelectric materials, respectively.

\section{AISb/InAs sample design and predicted band structure}

To test this concept, we have decided to use a simple material system. We use p-type InAs for the main semiconductor and p-type AlSb layers to modify the Seebeck coefficient. InAs and AlSb have been researched in depth by MBE before ${ }^{10}$ because the semiconductors are closely 
lattice-matched and have very different band gap energies. As seen in Figure 2, the band gap of $\mathrm{AlSb}$ is $\sim 1.55 \mathrm{eV}$ and InAs is $\sim 0.36 \mathrm{eV}$ at $300 \mathrm{~K}$.

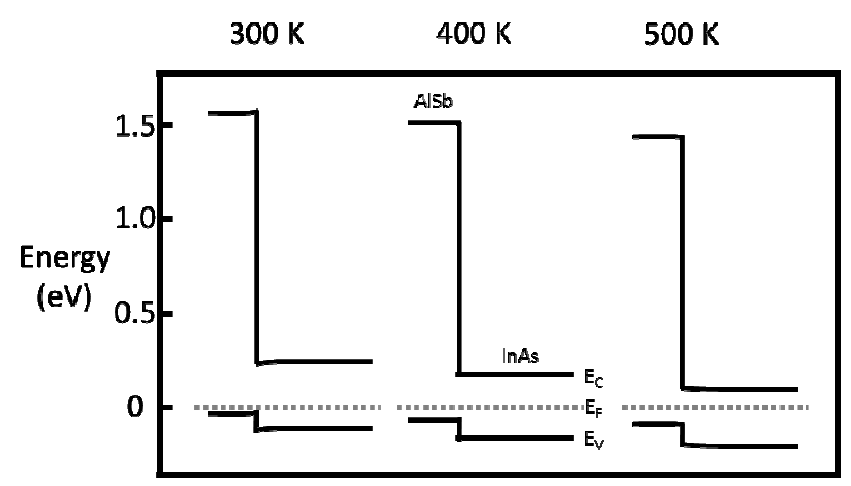

Figure 2. Calculated heterojunction between lightly p-doped InAs and heavily p-doped AlSb, at 300, 400, and 500 K. The Fermi level depends highly on temperature, especially for small band gap materials.

The valence band offset between $\mathrm{AlSb}$ and InAs is only $\sim 0.15 \mathrm{eV}$ while the conduction band offset is $\sim 1.35 \mathrm{eV}$. This combination of band offsets applies itself well to blocking the flow of electrons perpendicular to the heterojunction interface. InAs/AlSb is also a good model system because the "roll-over" in Seebeck coefficient should occur at relatively low temperatures and difficult substrate removal and layer encapsulation ${ }^{11}$ should not be necessary at the relevant measuring temperatures. The only disadvantage to using a small band gap semiconductor like InAs is the presence of a surface accumulation layer, ${ }^{12-15}$ which is caused by Fermi level surface pinning above the conduction band edge. This additional thin degenerate electron layer will be evident and noted in the measurement results.

\section{Sample fabrication}

Two samples were grown by molecular beam epitaxy, each having the same sample structure, but different Be doping concentrations in the active layer. Ideally, AlSb electron blocking layers would be used throughout the entire composite, but for MBE grown samples, the transport properties of that superlattice would have to be measured cross-plane, ${ }^{16}$ which is 
inherently difficult for electrical conductivity and Seebeck coefficient. For this study, we used a simple structure, which could be measured in-plane. We grew a Be-doped $2 \mu \mathrm{m}$-thick InAs layer with a $300 \mathrm{~nm}$-thick AlSb buffer on a (100) semi-insulating GaAs substrate. The GaAs substrate was used because it is cheaper and electrically insulating at room temperature. On top of the Bedoped InAs layer, we grew a thin 5 nm-thick heavily Be-doped InAs layer, a heavily Be-doped 500 nm-thick AlSb layer, and a heavily Be-doped 400 nm-thick InAs cap layer. The "heavy" doping in each of these thinner layers was $\sim 2 \times 10^{-19} \mathrm{~cm}^{-3}$. The band diagram, calculated using a Poisson-Schrodinger solver, is shown in Figure 3.
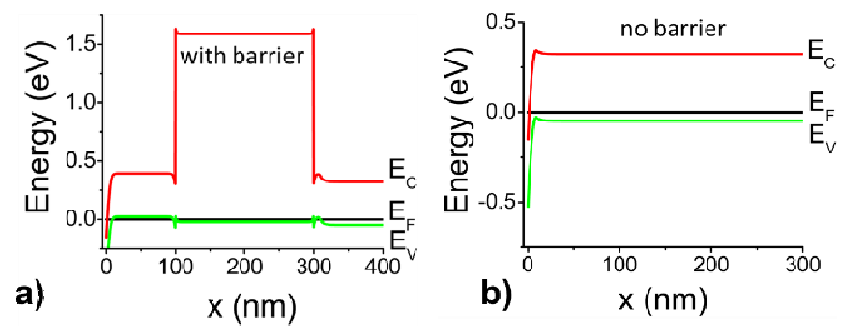

Figure 3. Band diagram for a) $\mathrm{p}+\mathrm{InAs} / \mathrm{p}+\mathrm{AlSb} / \mathrm{p}+\mathrm{InAs} / \mathrm{pInAs}$ which should show enhanced Seebeck coefficient due to minority carrier blocking, and b) p+InAs/pInAs, which should not show enhancement.

To utilize the minority carrier barriers in the intended manner, the samples were processed so that the AlSb barriers were only at the metal contacts, with the main InAs layer measured inplane between two heater blocks, as shown in Figure 4. The sample without AlSb barrier layers is exactly the same but without the top InAs or AlSb layer.

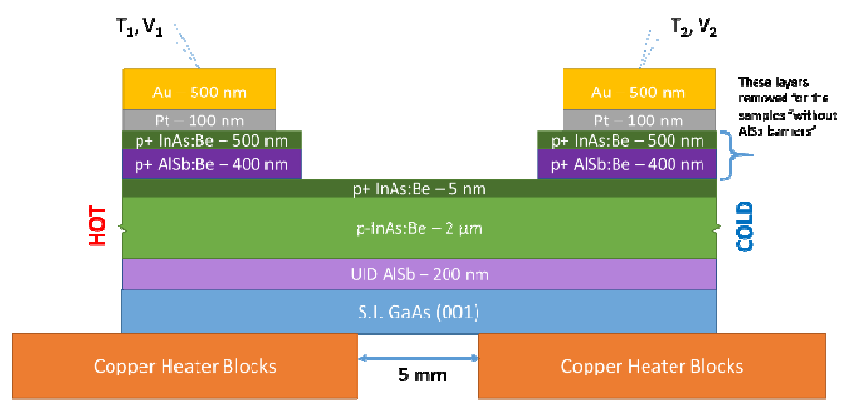

Figure 4. Cartoon schematic for the finished InAs sample with AlSb barrier layers, as it might be measured. Note that the layer thicknesses are not to scale relative to each other. 
To make this structure, the MBE-grown films were fabricated with typical semiconductor processing tools. The metal stack (100 nm Pt, $500 \mathrm{~nm} \mathrm{Au})$ was defined lithographically and then deposited using an e-beam evaporator. Pt was used because of its fairly large work function, which aligns well with the InAs valence band edge, and because it is relatively stable to high temperatures. Then the metal contacts and a very thin perimeter were covered with photoresist, again using photolithography. The InAs exposed region between contacts was wet etched with a solution of $\mathrm{H}_{2} \mathrm{O}: \mathrm{H}_{2} \mathrm{O}_{2}: \mathrm{H}_{3} \mathrm{PO}_{4}$ (concentration: 15:5:1 by volume), which selectively etches InAs over AlSb. Then the AlSb newly exposed region was wet etched with a solution of $\mathrm{H}_{2} \mathrm{O}: \mathrm{H}_{2} \mathrm{O}_{2}: \mathrm{HCl}$ (concentration: 100:1:100 by volume), which selectively etches AlSb over InAs.

The Seebeck coefficients were measured from a linear fit of voltage versus temperature difference $^{17}$ at each temperature data point, in vacuum, from $300-600 \mathrm{~K}$. The electrical conductivity was measured using the 4-point probe method or the Van der Pauw method, also from $300-600 \mathrm{~K}$. From $100-400 \mathrm{~K}$, the commercially available thermal transport option (TTO) was used in a Quantum Design physical property measurement system (PPMS). At room temperature, Hall and Van der Pauw measurement were made to determine carrier density and mobility.

\section{Measurement results}

The temperature dependent Seebeck coefficient, electrical conductivity, and power factor are shown in Figure 5 for two pairs of samples. In Figure 5a, we show the results for the high Be doping sample $\left(\sim 3 \times 10^{18} \mathrm{Be}\right.$ atoms per $\left.\mathrm{cm}^{3}\right)$, with and without AlSb barrier layers. The Seebeck coefficient and electrical conductivity for both samples is similar at $300 \mathrm{~K}$, but from $350-500$ $\mathrm{K}$, the Seebeck coefficient is higher for the sample with the AlSb barrier layers, by up to 50 $\mu \mathrm{V} / \mathrm{K}$. This results in almost a $100 \%$ increase in power factor at $425 \mathrm{~K}$, which is quite remarkable. In Figure 5b, we show the results for the low Be-doped sample $\left(\sim 1 \times 10^{18}\right.$ Be atoms per $\mathrm{cm}^{3}$ ), with and without AlSb barrier layers. For this Be doping, the Seebeck coefficient is almost $100 \mu \mathrm{V} / \mathrm{K}$ higher from $200-400 \mathrm{~K}$. For this temperature range, the difference in electrical conductivity of the samples with and without AlSb barriers is minor. This results in an increase in power factor of $150 \mathrm{~W} / \mathrm{mK}^{2}$, an increase of $200 \%$. 

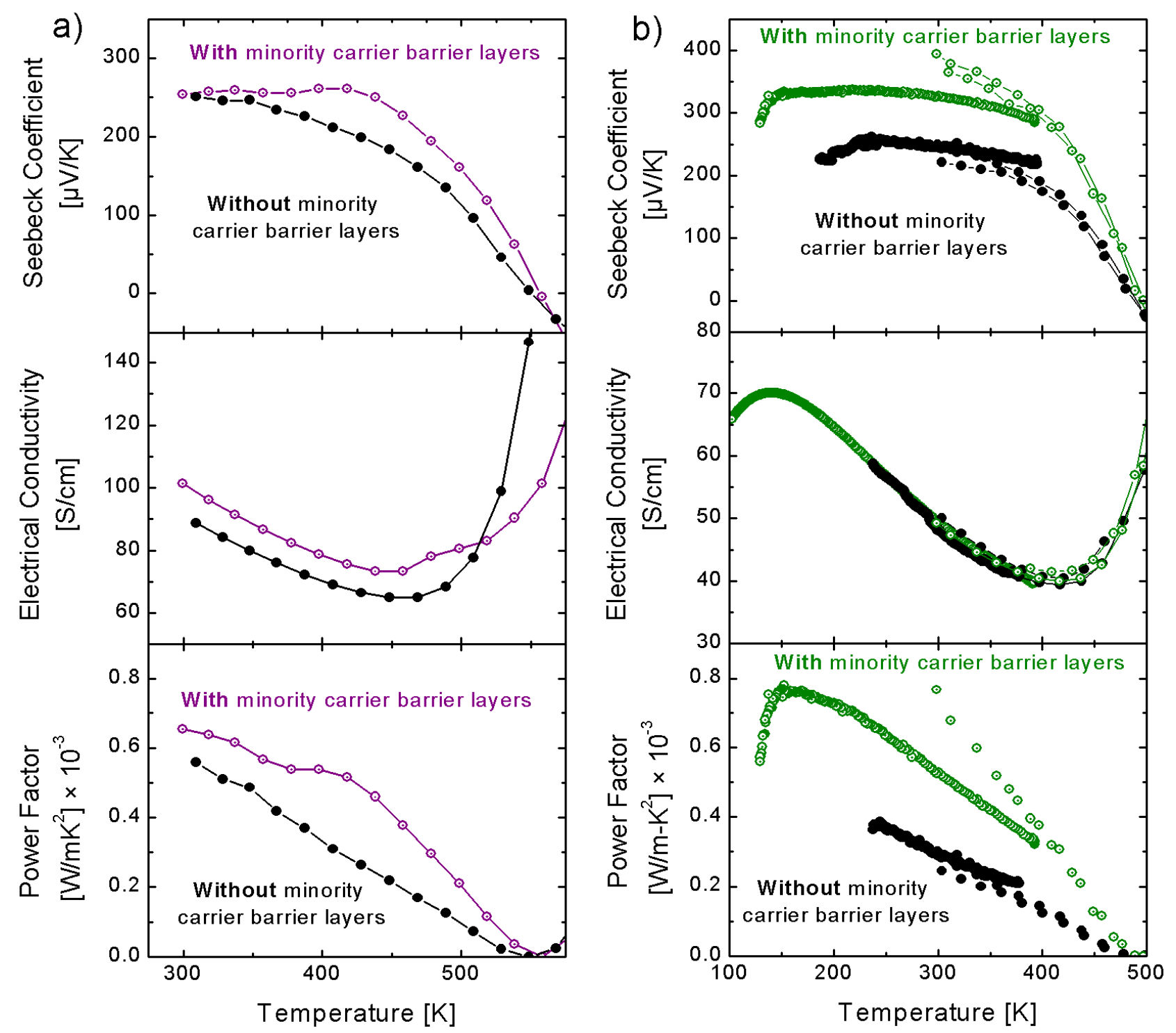

Figure 5. Measurement of increased Seebeck coefficient due to incorporation of minority carrier barriers. a) InAs doped with $\sim 3 \times 10^{18} \mathrm{Be}$ atoms $/ \mathrm{cm}^{3}$, and b) InAs doped with $\sim 1 \times 10^{18} \mathrm{Be}$ atoms $/ \mathrm{cm}^{3}$. In (b) the Seebeck data from $100 \mathrm{~K}-400 \mathrm{~K}$ was measured using the thermal transport option on a Quantum Design PPMS, while the Seebeck data from $300 \mathrm{~K}-500 \mathrm{~K}$ was measured under vacuum on a calibrated home-built system. Each measurement used a separate device fabricated from the same wafer. 
To better understand the effect of the AlSb electron blocking layers, we conducted Van der Pauw and Hall measurements, which are shown in Table I. Again we note that the conductivities of the samples do not change dramatically with and without AlSb barriers. It is remarkable, yet not unexpected, that the Hall coefficient becomes more positive for both pairs of InAs samples, with and without barriers, suggesting that a higher hole concentration is being measured when AlSb barriers are present.

Table I. Room temperature carrier density and mobility of Be-doped InAs with and and without AlSb barriers.

\begin{tabular}{cccccc} 
& & $\begin{array}{c}\text { Electrical } \\
\text { Conductivity } \\
(\mathrm{S} / \mathrm{cm})\end{array}$ & $\begin{array}{c}\text { Hall } \\
\text { Coefficient } \\
\left(\mathrm{cm}^{3} / \mathrm{C}\right)\end{array}$ & $\begin{array}{c}\text { Carrier } \\
\text { Density } \\
\left(\mathrm{cm}^{-3}\right)\end{array}$ & $\begin{array}{c}\text { Mobility } \\
\left(\mathrm{cm}^{2} / \mathrm{V}-\mathrm{s}\right)\end{array}$ \\
\cline { 3 - 6 } High Be doping & With AlSb barrier & 97.79 & $4.19 \times 10^{-5}$ & $7.45 \times 10^{18}$ & 82 \\
& Without AlSb barrier & 80.41 & $1.07 \times 10^{-4}$ & $2.90 \times 10^{18}$ & 172 \\
\cline { 2 - 5 } Low Be doping & With AlSb barrier & 49.4 & $-5.80 \times 10^{-4}$ & $-5.38 \times 10^{17}$ & -573 \\
& Without AlSb barrier & 47.18 & $-1.23 \times 10^{-5}$ & $-2.54 \times 10^{19}$ & -12
\end{tabular}

\section{Analysis and discussion}

The Seebeck coefficient and electrical conductivity were calculated in the manner described by Wagener et $a l^{18}$ accounting for the degenerate and non-degenerate regimes. These fits are shown in Figure 6 and Figure 7 by the dotted red lines. The model was then adjusted to remove the effect of the n-type surface accumulation layer and to lower the number of thermally activated minority carriers. These results are represented by the black line in Figure 6 and Figure 7. We use the input of acceptor concentrations and the ratio of electron mobility to hole mobility to fit our data, with good agreement. The increase in Seebeck coefficient is greater for the InAs samples with less Be doping, which intuitively makes sense. For example, if the extrinsic doping is lower, the thermally activated minority carriers will be more detrimental. By removing their input on Seebeck coefficient, we see a bigger increase. This is further proof that the increase in Seebeck coefficient is due to electron blocking in these samples. 


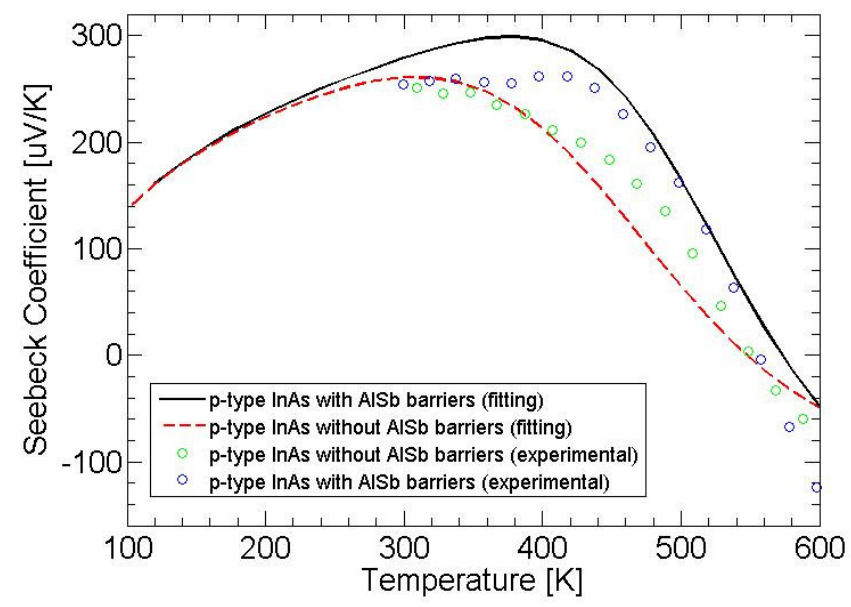

Figure 6. Fitted experimental data for highly Be-doped InAs. The dotted red line and green circles represent typical InAs, accounting for the surface accumulation layer. The black line and blue circles represent InAs with relatively fewer minority (holes) carriers and no surface accumulation layer contribution.

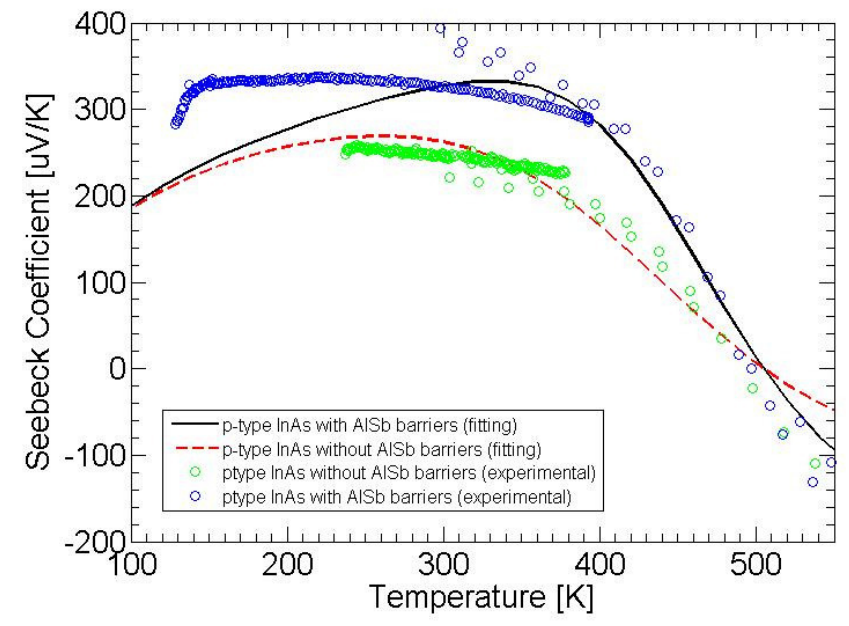

Figure 7. Fitted experimental data for low Be-doped InAs. The dotted red line and green circles represent typical InAs, accounting for the surface accumulation layer. The black line and blue circles represent InAs with relatively fewer minority (holes) carriers and no surface accumulation layer contribution 
Intuitively, blocking one type of carrier should lower the total electrical conductivity of the system, everything else being equal. Our calculations show us that this is true, but not to the extent we would expect. For instance, in Figure 8, we calculate that the electrical conductivity only changes by $\sim 5 \mathrm{~S} / \mathrm{cm}$ even when the minority carrier barrier is most pronounced. These calculations are under the same conditions to fit the high Be doped InAs Seebeck coefficient.

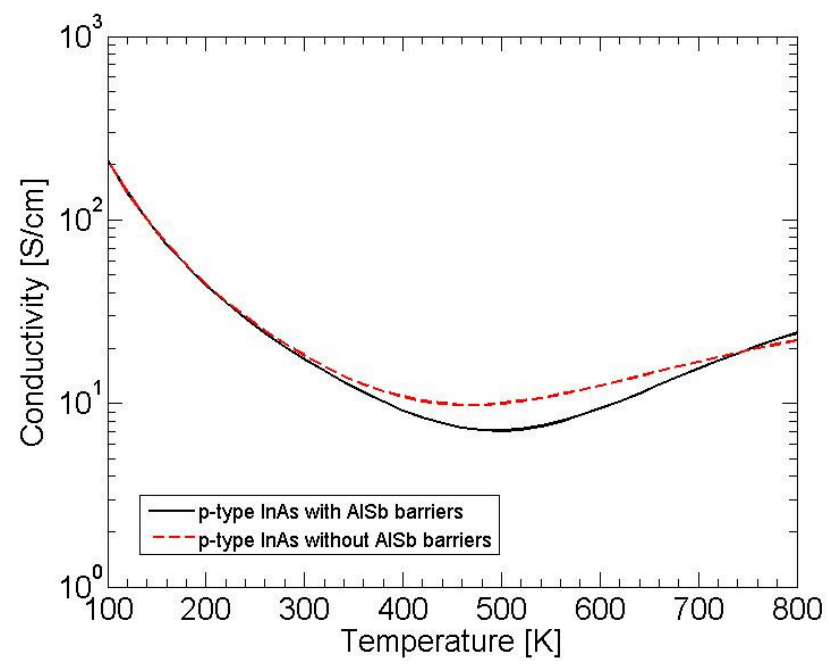

Figure 8. Theoretical electrical conductivity for p-type InAs, a) accounting for the surface accumulation layer and intrinsic carriers (red dotted line) and b) assuming no surface accumulation layer and fewer minority intrinsic carriers (black line). Of note is the relative similarity between both simulations.

\section{Conclusion}

We have conceived and demonstrated an idea for improving Seebeck coefficient and power factor in semiconductor materials using careful band engineering. This solution addresses the problem of bipolar conductivity, which occurs in semiconductors at high temperature. When the concentration of thermally activated intrinsic carriers becomes appreciable, the Seebeck coefficient decreases and the thermal conductivity increases. We have shown that inserting wide band gap semiconductor layers in a narrow band gap semiconductor host can improve the Seebeck coefficient by blocking minority carriers. This idea increases the thermoelectric power factor by $2 \times$ to $2.5 \times$ in our model system on p-type InAs with p+AlSb electron blocking layers. Additionally, using a superlattice should lower the thermal conductivity. ${ }^{8}$ In short, accounting for and managing the minority carrier transport should increase the thermoelectric figure of merit 
by simultaneously increasing the power factor and reducing thermal conductivity. The first steps to proving this concept have been demonstrated here.

\section{Acknowledgements}

The authors thank Je-Hyeong Bahk and Ali Shakouri, at the University of Purdue for helpful discussion on heterojunction modeling. The authors also thank John English and Hong Lu for help with the MBE growth. This work was supported through the Center for Energy Efficient Materials, an Energy Frontier Research Center funded by the U.S. Department of Energy, Office of Science, Office of Basic Energy Sciences under Award Number DE-SC0001009. Additionally, the low temperature measurements were conducted in the MRL Shared Experimental Facilities, which are supported by the MRSEC Program of the NSF under Award No. DMR 1121053; a member of the NSF-funded Materials Research Facilities Network (www.mrfn.org).

\section{References}

1. Stabler, F. R. "Commercialization of thermoelectric technology." in Mat. Res. Soc. Symp. Proc. Vol. 886 886, 0886-F01-04.1 (Warrendale, Pa.; Materials Research Society; 1999, 2005).

2. Kajikawa, T. "Approach to the practical use of thermoelectric power generation." $J$. Electron. Mater. 38, 1083-1088 (2009). doi:10.1007/s11664-009-0831-2

3. Sofo, J. O. \& Mahan, G. D. "Optimum band gap of a thermoelectric material." Phys. Rev. $B$ 49, 4565-4570 (1994).

4. Mahan, G. D. \& Sofo, J. O. "The best thermoelectric." Proc. Natl. Acad. Sci. U.S.A. 93, 7436 (1996).

5. Gautam, N., Kim, H. S., Kutty, M. N., Plis, E., Dawson, L. R. \& Krishna, S. "Performance improvement of longwave infrared photodetector based on type-II InAs/GaSb superlattices using unipolar current blocking layers." Appl. Phys. Lett. 96, 231107 (2010). doi:10.1063/1.3446967

6. Gautam, N., Myers, S., Barve, A. V., Klein, B., Smith, E. P., Rhiger, D. R., Dawson, L. R. \& Krishna, S. "High operating temperature interband cascade midwave infrared detector based on type-II InAs/GaSb strained layer superlattice." Appl. Phys. Lett. 101, 021106 (2012). doi:10.1063/1.4733660 
7. Maimon, S. \& Wicks, G. W. "nBn detector, an infrared detector with reduced dark current and higher operating temperature." Appl. Phys. Lett. 89, 151109 (2006).

doi:10.1063/1.2360235

8. Bahk, J. H. \& Shakouri, A. "Enhancing the thermoelectric figure of merit through the reduction of bipolar thermal conductivity with heterostructure barriers." Appl. Phys. Lett. 105, 052106 (2014). doi:10.1063/1.4892653

9. Tynell, T., Giri, A., Gaskins, J., Hopkins, P. E., Mele, P., Miyazaki, K. \& Karppinen, M. "Efficiently suppressed thermal conductivity in $\mathrm{ZnO}$ thin films via periodic introduction of organic layers." J. Mater. Chem. A 2, 12150-12152 (2014). doi:10.1039/c4ta02381a

10. Kroemer, H. "The $6.1 \AA$ family (InAs, GaSb, AlSb) and its heterostructures: a selective review." Phys. E Low-dimensional Syst. Nanostructures 20, 196-203 (2004).

doi:10.1016/j.physe.2003.08.003

11. Bahk, J.-H., Zeng, G., Zide, J. M. O., Lu, H., Singh, R., Liang, D., Ramu, A. T., Burke, P. G., Bian, Z., Gossard, A. C., Shakouri, A. \& Bowers, J. E. "High-Temperature Thermoelectric Characterization of III-V Semiconductor Thin Films by Oxide Bonding." J. Electron. Mater. 39, 1125-1132 (2010). doi:10.1007/s11664-010-1258-5

12. Noguchi, M., Hirakawa, K. \& Ikoma, T. "Intrinsic electron accumulation layers on reconstructed clean InAs(100) surfaces." Phys. Rev. Lett. 66, 2243-2246 (1991). doi:10.1103/PhysRevLett.66.2243

13. Olsson, L., Andersson, C., Håkansson, M., Kanski, J., Ilver, L. \& Karlsson, U. "Charge Accumulation at InAs Surfaces." Phys. Rev. Lett. 76, 3626-3629 (1996). doi:10.1103/PhysRevLett.76.3626

14. Tsui, D. "Observation of Surface Bound State and Two-Dimensional Energy Band by Electron Tunneling." Phys. Rev. Lett. 24, 303-306 (1970). doi:10.1103/PhysRevLett.24.303

15. Piper, L., Veal, T., Lowe, M. \& McConville, C. "Electron depletion at InAs free surfaces: Doping-induced acceptorlike gap states." Phys. Rev. B 73, 195321 (2006). doi:10.1103/PhysRevB.73.195321

16. Zeng, G., Zide, J. M. O., Kim, W., Bowers, J. E., Gossard, A. C., Bian, Z. X., Zhang, Y., Shakouri, A., Singer, S. L. \& Majumdar, A. "Cross-plane Seebeck coefficient of ErAs:InGaAs/InGaAlAs superlattices." J. Appl. Phys. 101, 034502 (2007). doi:10.1063/1.2433751

17. De Boor, J. \& Mu $\square$ ller, E. "Data analysis for Seebeck coefficient measurements." Rev. Sci. Instrum. 84, 065102 (2013). doi:10.1063/1.4807697 
18. Wagener, M. C., Wagener, V. \& Botha, J. R. "Thermoelectric evaluation of the dopant density of p-type InAs." Appl. Phys. Lett. 94, 262106 (2009). doi:10.1063/1.3167822 